\title{
Short communication: Influence of long-chain inulin and Lactobacillus paracasei subspecies paracasei on the sensory profile and acceptance of a traditional yogurt
}

\author{
T. C. Pimentel, ${ }^{*}$ A. G. Cruz,† and S. H. Prudencio*1 \\ *Universidade Estadual de Londrina (UEL), Departamento de Ciência e Tecnologia de Alimentos (DCTA), Londrina, Paraná, 86051-970, Brazil \\ †Instituto Federal de Educação, Ciência e Tecnologia do Rio de Janeiro (IFRJ), Mestrado Profissional em Ciência e Tecnologia de Alimentos \\ (PGCTA), Rua Senador Furtado, 171, Maracanã, 20270-021, Rio de Janeiro, Brazil
}

\begin{abstract}
The objectives of this study were to evaluate the influence of the addition of long-chain inulin as a fat replacer and prebiotic agent $(20 \mathrm{~g} / \mathrm{L})$ and (or) probiotic Lactobacillus paracasei ssp. paracasei on the sensory profile and acceptance of yogurts, and to assess the influence of descriptive attributes on the sensory acceptance of the products. The addition of inulin to low-fat yogurt improved its brightness and firmness, which was similar to the full-fat yogurt. However, the use of longchain inulin increased the separation of serum and no influence on creaminess was observed. Regarding the product's acceptability, the low-fat yogurt with added inulin presented similar acceptance compared with the full-fat yogurt. The addition of $L b$. paracasei ssp. paracasei did not affect the sensory profile and acceptance of the low-fat yogurt. Using external preference mapping, it was possible to verify that the sensory acceptance was driven positively by the sweetness and creaminess and negatively driven by firmness (appearance and texture) and homogeneity (appearance). It was possible to formulate low-fat yogurts with added probiotics that presented similar sensory characteristics to those of full-fat yogurts, and this was due to the addition of the long-chain inulin as a fat replacer.
\end{abstract}

Key words: prebiotic, probiotic, sensory profiling, fat replacer

\section{Short Communication}

Probiotics are live microorganisms that confer a beneficial effect on the host when administered in adequate amounts (FAO/WHO, 2001). Many health benefits have been associated with the consumption of Lactobacillus paracasei ssp. paracasei (Lb. casei 01), including antimicrobial activity; antimutagenic, anticarcinogenic,

Received February 14, 2013.

Accepted June 28, 2013.

${ }^{1}$ Correponding author: sandrah@uel.br and antioxidant properties; and stimulation of the immune system (Mirzaei et al., 2008; Tharmaraj and Shah, 2009; Liu et al., 2011).

Prebiotics are nonviable food components that provide health benefits to the host, associated with modulation of the microbiota (FAO/AGNS, 2007). Inulintype fructans are the main compounds with prebiotic properties. The degree of polymerization (DP) represents the number of monosaccharides that comprise the molecule and can influence some properties of these ingredients, such as digestibility, prebiotic activity, sweetness, and water-holding capacity, among others (Pimentel et al., 2012a). Medium-length inulin presents a DP of 10, whereas long-chain inulin (DP 23) is obtained by physical removal of short-chain molecules. Oligofructoses are fructan-type inulins with a DP $<10$ and are produced by partial hydrolysis of inulin (Meyer et al., 2011a; Cruz et al., 2013). The consumption of long-chain inulin has been linked to health benefits such as inhibition of growth of pathogens in the gut (Patterson et al., 2010), enhanced calcium absorption from the diet (Coudray et al., 2005), relief of constipation (Den Hond et al., 2000), decrease in blood lipid concentrations (Cieslik et al., 2005), stimulation of the immune system (Ryz et al., 2009), and anticarcinogenic properties (Hughes and Rowland, 2001).

The technological use of inulin is based on its properties: sugar replacer (especially in combination with sweeteners), fat replacer, and texture modifier. As a fat replacer in low-fat dairy products, inulin is a suitable option because it has the ability to form microcrystals when mixed with water or milk. These crystals are not perceived in the mouth, but interact with the food matrix to form a finely creamy texture that promotes an oral tactile sensation comparable with that of fat (Franck, 2002; Meyer et al., 2011a).

Long-chain inulin is more stable, less soluble, and more viscous than the native inulin (Wada et al., 2005), and presents a high capacity to replace fat in many types of food products, such as yogurts (Paseephol et al., 2008; Guggisberg et al., 2009; Modzelewska-Kapituła 
and Kłębukowska, 2011), dairy beverages (Villegas et al., 2007), cheeses with reduced fat content (Koca and Metin, 2004; Hennelly et al., 2006), ice cream (Akalin and Erişir, 2008; Karaca et al., 2009), dairy desserts (Gonzalez-Tomás et al., 2008; Torres et al., 2010), nondairy soy-based desserts (Granato et al., 2010), and skim milk (Meyer et al. 2011b).

No report exists in the literature that evaluates, concomitantly, the effect of adding long-chain inulin as a fat replacer and prebiotic agent, a probiotic, or a combination of these functional components on the sensory properties of yogurt. Thus, the objectives of this study were to evaluate the influence of the addition of long-chain inulin as a fat replacer and prebiotic and (or) probiotic Lactobacillus paracasei ssp. paracasei on the sensory profile and acceptance of yogurts and to assess the influence of descriptive attributes on the sensory acceptance of the products.

Five formulations of yogurts were prepared according to Pimentel et al. (2012b), where one sample was traditional full-fat and the remaining samples were low-fat yogurts: traditional, prebiotic (with added long-chain inulin); probiotic (with added Lactobacillus paracasei ssp. paracasei), and synbiotic (with added long-chain inulin and Lactobacillus paracasei ssp. paracasei). Powdered skim or whole milk (Nestlé, São Paulo, Brazil) was reconstituted to $130 \mathrm{~g} / \mathrm{L}$ with distilled water. To standardize the TS, in the formulations without inulin addition (Raftiline HP; Orafti SA, Oreye, Belgium; average DP of 23), a total of $35 \mathrm{~g} / \mathrm{L}$ of skim milk powder was added to the formulations, whereas samples that contained inulin had $15 \mathrm{~g} / \mathrm{L}$ of skim milk powder and $20 \mathrm{~g} / \mathrm{L}$ of long-chain inulin added to them (Akalın et al., 2004). The mixtures were pasteurized at $85^{\circ} \mathrm{C}$ for 30 min and cooled using an ice bath to $42^{\circ} \mathrm{C}$ (Akalın et al. 2004). The starter culture (Lactobacillus delbrueckii ssp. bulgaricus and Streptococcus thermophilus) package content (YC X-11; Chr. Hansen, São Paulo, Brazil) was poured into $1 \mathrm{~L}$ of sterilized milk, mixed, and stored frozen (50-mL aliquots). For every yogurt processing, it was thawed and inoculated in a quantity of $2 \mathrm{~mL} / \mathrm{L}$ with or without the addition of $0.1 \mathrm{~g} / \mathrm{L}$ of Lactobacillus paracasei ssp. paracasei (L. casei 01; Chr. Hansen, Valinhos, Brazil), which corresponded to $10^{11}$ viable cells in 1 $\mathrm{L}$ of the final product. The cultures were used according to manufacturer's instructions. The inoculated products were placed in sterile plastic containers with a lid and a capacity of $80 \mathrm{~mL}$ and incubated at $42^{\circ} \mathrm{C}$ until a $\mathrm{pH}$ of 4.5 was reached. Then, the products were cooled to $4^{\circ} \mathrm{C}$ (Donkor et al., 2007) for $1 \mathrm{~d}$ and analyzed.

The sensory profile was established by using quantitative descriptive analysis (QDA; Gonzalez et al., 2011; Akalın et al., 2012; Kaaki et al., 2012). Twenty-five individuals, who were regular consumers of yogurts, were selected among students and staff members from the State University of Londrina (Londrina, Paraná, Brazil). Initially, the consumers were subjected to a test to recognize basic tastes and odors (Meilgaard et al., 1998). Individuals who could not identify at least 1 of each of the basic tastes or did not achieve the minimum of $60 \%$ accuracy in basic odors were excluded from the sensory panel. The development of the descriptive terminology by the selected panelists was carried out using the network method (Moskowitz, 1983). The descriptors (attributes), definitions, and reference samples developed by the sensory panel are shown in Table 1. The evaluation sheet was prepared in consensus and was composed of a 9-cm unstructured scale, and the lines had anchor descriptors at $0.5 \mathrm{~cm}$ from the ends, in which the left anchor represented the lowest intensity.

The panelists were trained to identify and quantify the attributes using the reference samples (Table 1). After 8 training sessions, the performance of each panelist was assessed. Three formulations (full-fat, traditional low-fat, and synbiotic) were evaluated using a randomized complete block design containing 3 replications. The intensity of the attributes was assessed using the evaluation sheet. Data from each panelist were evaluated using 2-factor ANOVA (samples and replicates) and F-test.

A total of 12 trained panelists (9 women and 3 men) were selected for presenting discriminative capacity $(P$ value of $\left.\mathrm{F}_{\text {formulations }}<0.5\right)$, reproducibility ( $P$-value of $\left.\mathrm{F}_{\text {repetitions }} \geq 0.05\right)$, where $\mathrm{F}$ is the parameter of the $\mathrm{F}$ test, and consensus with other members of the group (Damasio and Costell, 1991). About 50\% of the panelists were aged 15 to $25 \mathrm{yr}$ old, $41.67 \%$ were between 25 and $35 \mathrm{yr}$ old, and $8.33 \%$ were between 35 and $50 \mathrm{yr}$ old.

The descriptive analysis of the 5 formulations performed by trained judges was carried out in triplicate, where each panelist evaluated each formulation in each section, using the evaluation sheet developed during the training sessions. For all formulations, the aroma was the first property assessed and then the appearance, flavor, and texture were evaluated afterward.

The acceptability of the formulations was evaluated by 75 potential consumers of the products (Drake, 2007; Cruz et al., 2012b) through a hedonic scale of 9 points $(9=$ like extremely; $1=$ dislike extremely). Fifty milliliters of the formulations, in their original containers, coded with 3 -digit random numbers and at $4^{\circ} \mathrm{C}$, were evaluated in individual booths in a monadic way and in random order, under white light.

The consumers were recruited orally based on their availability to participate in the test and if they were consumers of yogurt. Regarding the frequency of con- 
Table 1. Attributes and definitions developed by the descriptive sensory panel

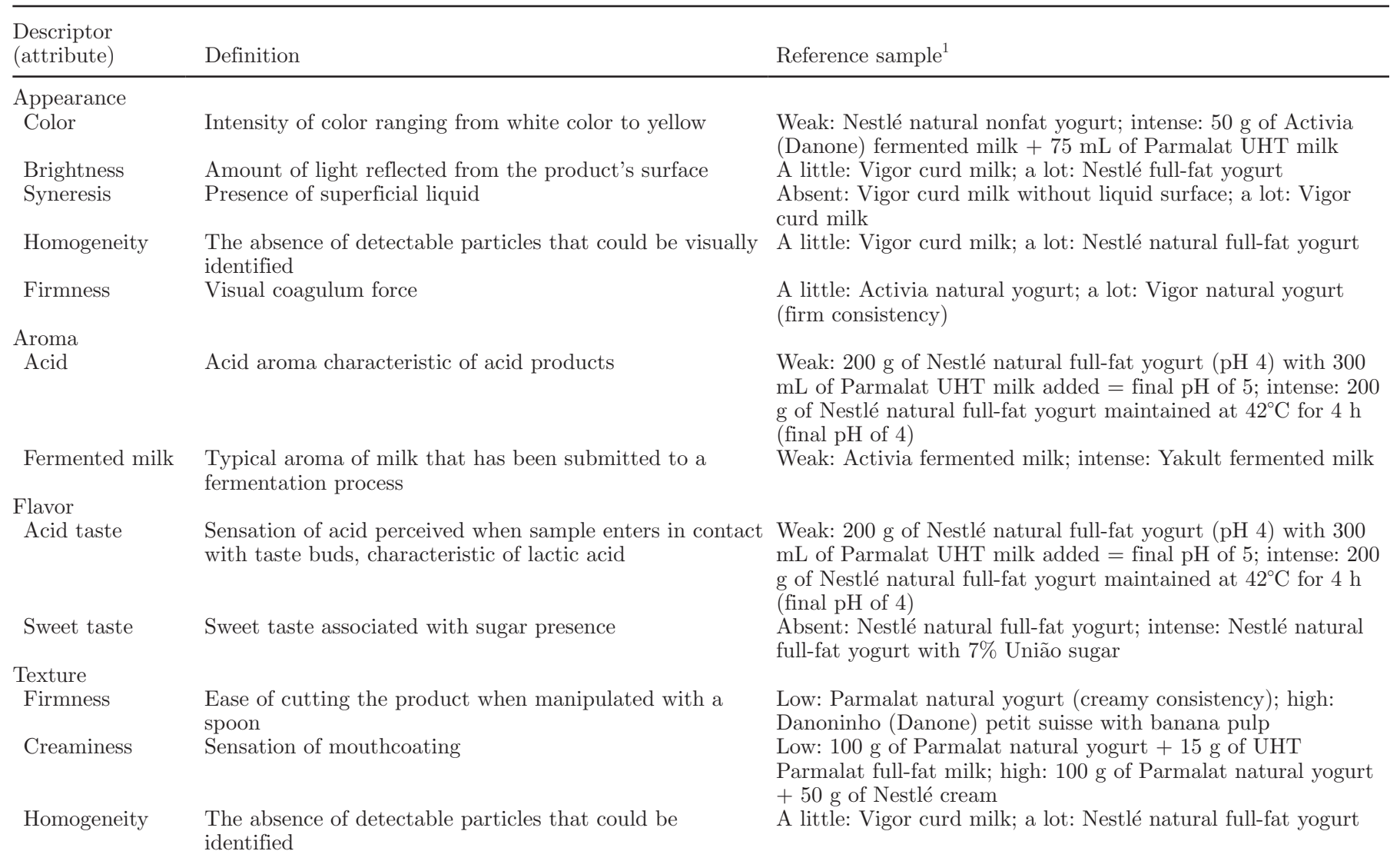

${ }^{1}$ Nestlé yogurt (Nestlé, São Paulo, Brazil); Activia products (Danone Brasil, São Paulo, Brazil); Parmalat Brasil S/A (São Paulo, Brazil); Vigor products (Vigor SA, São Paulo, Brazil); Yakult fermented milk (Yakult SA, São Paulo, Brazil); União sugar (União, Araquari, Santa Catarina, Brazil); Danoninho petit suisse (Danone Brasil).

sumption of plain yogurt, $38.46 \%$ of the consumers drank it moderately (a few times per month), $28.21 \%$ drank it often (several times per week) and $26.92 \%$ drank it occasionally (a few times per year). Only $6.41 \%$ of the consumers reported never consuming this type of yogurt.

Four liters of each formulation were made for each trial, totaling 4 replications in all experiments, 3 for the QDA test, and 1 for the consumer test. A randomized complete block design was used, with treatments being the formulations and the blocks being the judges. The QDA experiment was repeated 3 times. Data were submitted to ANOVA, F-test, the Tukey comparison of the means test, and principal components analysis (PCA). A significance level of $P \leq 0.05$ was used. External preference mapping (Cadena et al., 2012) was conducted by first using PCA on the descriptive sensory analysis data and then relating each of the consumers to this PCA space by regression analysis. Statistical analyzes were conducted in SAS software (version 9.1.3; SAS Institute Inc., Cary, NC) and XLSTAT for Windows (version 2012; Addinsoft, New York, NY).
When the data from the QDA was subjected to PCA, it was possible to observe that the first 3 components presented eigenvalues higher than 1 and should be interpreted using the Kaiser criterion (Lawless and Heymann, 1998). The first principal component (PC1) explained $53.16 \%$ of the variability contained in the original variables, whereas the second (PC2) and third principal components (PC3) explained 15.92 and $11.96 \%$, respectively, totaling $81 \%$ of the explained variability in the data matrix.

In each PC, the attributes that presented a correlation coefficient $\geq \pm 0.6$ were considered important (Chapman et al., 2001; Hair et al., 2010). The following variables correlated to $\mathrm{PC} 1$ in descending order: homogeneity (appearance), brightness, fermented milk aroma, acid aroma, firmness (texture), homogeneity (texture) and firmness (appearance; positive correlations), and creaminess and sweet taste (negative correlations). Color and syneresis were positively correlated with PC2. Acid taste was positively correlated and homogeneity (texture) was negatively correlated with PC3 (Figures 1A and 1B). 
Figure 1 contains the graphical representations from the PCA data. Each formulation is represented by 3 points, where each point corresponds to the mean value assigned by the sensory panel in each repetition. The points for the same formulation are close, thus demonstrating that good repeatability among the panelists. The formulations that were similar with respect to the attributes judged are close together and the different ones are far from one another. Principal component 1 separated the formulations in relation to the fat content (the left side presents the full-fat yogurt and the right side contains the low-fat samples: traditional, prebiotic, probiotic, and synbiotic). Principal component 2 separated the samples with added inulin; the traditional low-fat and the probiotic yogurt (without inulin) remained below the axis and the prebiotic and synbiotic yogurts (with inulin) remained above the axis, with the full-fat formulation closer to those added inulin (above the axis). Principal component 3 separated formulations by the presence of probiotic: without addition (traditional low-fat and prebiotic) below the axis and with addition (probiotic and synbiotic) above the axis, with the full-fat formulation closer to those added probiotic (above the axis). These results indicate that the fat content and the addition of inulin or probiotic to the yogurt resulted in different sensory characteristics.

Table 2 shows the mean values for the sensory attributes of each formulation, confirming some of the results obtained by PCA, taking on a significance level less than or equal to $5 \%$. The addition of a probiotic culture to the low-fat yogurt did not cause any perceived sensory changes, once no significant differences existed between the traditional low-fat and probiotic formulations in any attributes $(P>0.05$; Table 2$)$. The sensory results are consistent with those from physicochemical analyses, where no effect was observed of adding a probiotic strain on $\mathrm{pH}$, titratable acidity, color, and syneresis of yogurts (Pimentel et al., 2012b). The PCA graph suggests differences in the probiotic-added formulations (probiotic and synbiotic) compared with the traditional low-fat product, but these results were not confirmed by ANOVA $(P>0.05)$.

The prebiotic yogurt presented similar intensities in color, firmness (appearance and texture), acid taste, and sweet taste compared with the full-fat yogurt $(P>$ $0.05)$. Significant differences $(P \leq 0.05)$ were obtained between the prebiotic and the full-fat yogurts with respect to the other sensory features. When the prebiotic and the traditional low-fat yogurts were compared, only brightness and syneresis were different $(P \leq 0.05)$, and it was possible to observe that the addition of inulin made the low-fat yogurt become more shiny; however, the product presented more syneresis. Possibly, the inulin gel presented more brightness than the protein milk gel (casein), or the increased presence of serum could influence the perception of brightness, as larger amounts of serum produce greater light reflection. The presence of the long-chain inulin likely interfered with the development of a 3-dimensional structure of casein, leading to a weaker gel incapable of retaining water (Paseephol et al., 2008).

The low-fat traditional yogurt had higher firmness than the full-fat product $(P \leq 0.05)$, and the addition of inulin (prebiotic and synbiotic formulations) resulted in less-firm yogurt (assessed on the spoon test), which was similar to the full-fat product $(P>0.05)$. However, when firmness in the appearance of the yogurt was assessed, the results indicated no differences among formulations $(P>0.05)$. The molecules of inulin, as well as the fat globules, may be dispersed within the casein micelles, interfering with the formation of the protein matrix, resulting in a softer gel (Paseephol et al., 2008). The results for firmness indicate the possible role of inulin as a fat replacer, and confirm the results of firmness evaluated by the instrumental texture analysis (Pimentel et al., 2012b).

Yogurts with added inulin (prebiotic and synbiotic formulations) presented more intense homogeneous appearance, acid aroma, and fermented milk aroma compared with the traditional full-fat yogurt $(P \leq 0.05)$. However, these characteristics were similar to those of low-fat yogurts $(P>0.05)$. The results indicate that the addition of inulin does not change the acidity of low-fat yogurt as perceived by the taste and aroma of the products. The higher aroma acidity observed for the prebiotic yogurt compared with full-fat yogurt may be due to different chemical compositions of the prepared yogurts and was also observed in physicochemical analysis ( $\mathrm{pH}$ and titratable acidity; Pimentel et al., 2012b). Prebiotic yogurt had higher protein and lactose levels. The protein can interfere with the $\mathrm{pH}$ due to the buffering capacity of proteins, and the lactose is the preferred substrate used by microorganisms, leading to the formation of organic acids (Akalin et al., 2007).

Regarding the homogeneity of the test samples, the presence of fat in the full-fat formulation may have influenced the appearance and the oral sensation the product provides. When the milk was homogenized, the fat globules may have remained dispersed in yogurt, becoming easily visible and perceived during sensory evaluation. This technological problem may have diminished the acceptance of the product in relation to its appearance.

The full-fat formulation garnered higher scores for creaminess than the low-fat yogurts (traditional, prebiotic, probiotic, and synbiotic; $P \leq 0.05$ ), indicating that inulin had no effect on the creaminess. Possibly, inulin is capable of increasing the creaminess of yogurts 
A)

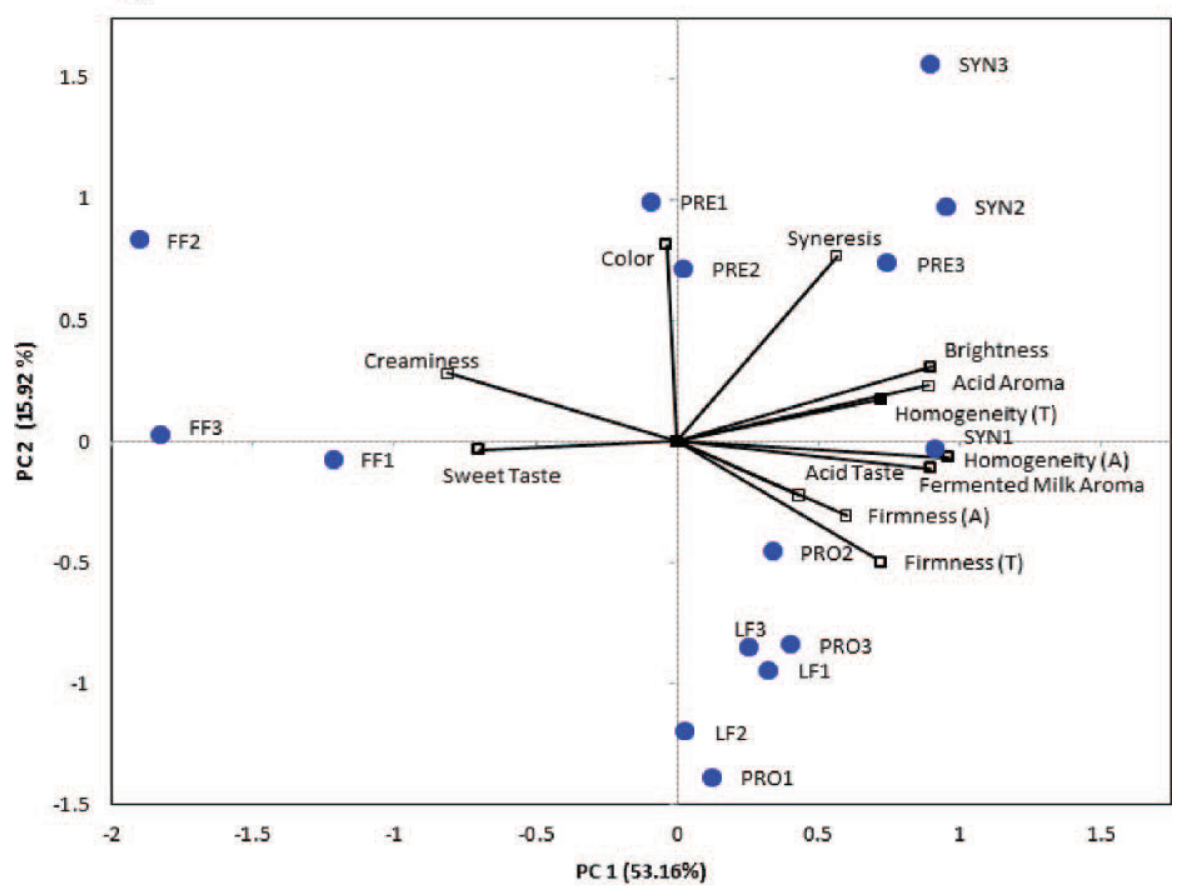

B)

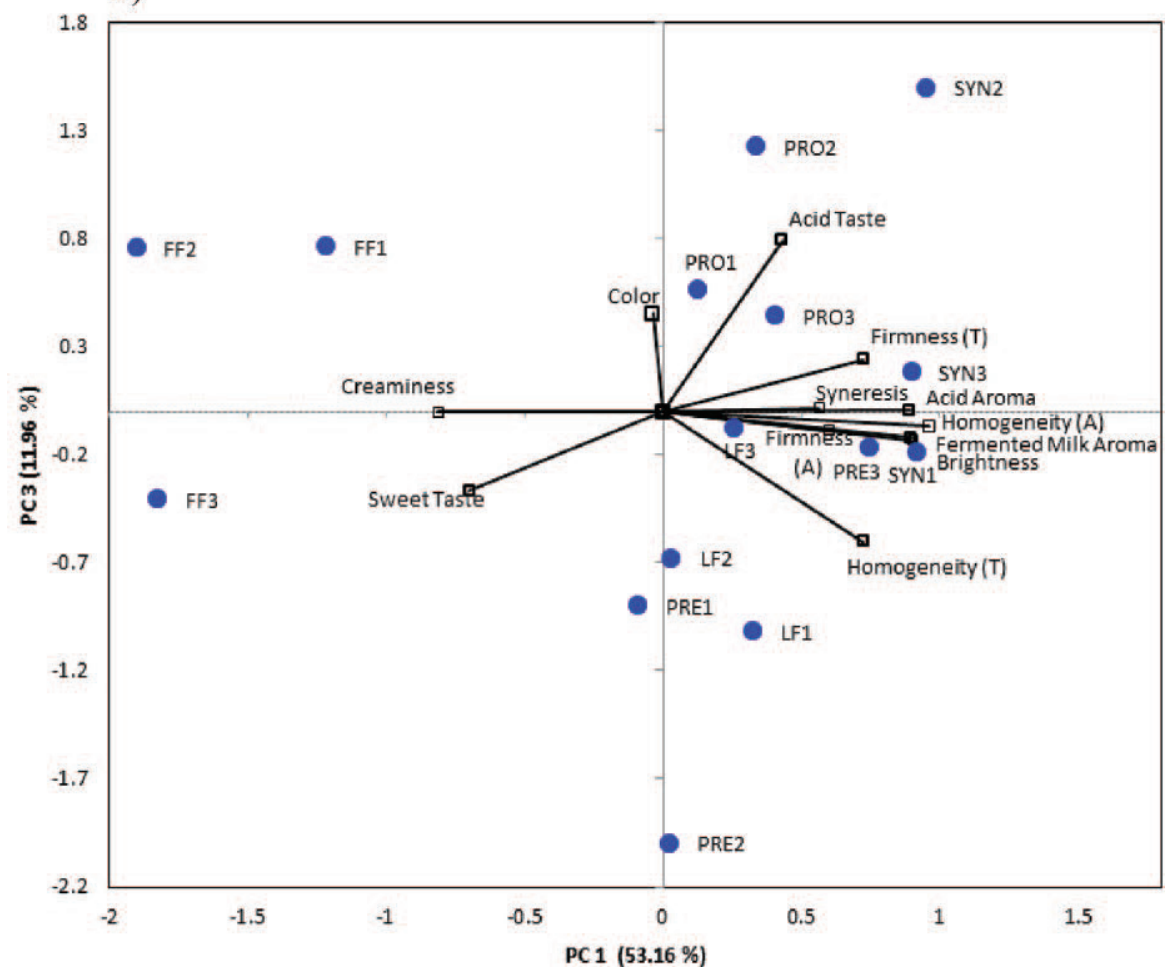

Figure 1. Sensory principal components (PC) plots: attributes (vectors) and formulations (circles). (A) Principal component 1 versus PC2; (B) PC1 versus PC3. Formulations and repetitions: FF1, FF2, and FF3 = full-fat yogurt; LF1, LF2, and LF3 = traditional low-fat yogurt; PRE1, PRE2, and PRE3 = prebiotic (low-fat yogurt with added inulin); PRO1, PRO2, PRO3 = probiotic (low-fat yogurt with added Lactobacillus paracasei ssp. paracasei); SYN1, SYN2, and SYN3 = synbiotic (low-fat yogurt with added inulin and Lactobacillus paracasei ssp. paracasei). An A in parentheses corresponds to attributes evaluated for appearance and a $\mathrm{T}$ in parentheses corresponds to attributes evaluated for texture. Color version available in the online PDF. 
Table 2. Mean intensity values of the quantitative descriptive analysis (QDA) attributes for each formulation

\begin{tabular}{|c|c|c|c|c|c|}
\hline \multirow[b]{2}{*}{ Attribute } & \multicolumn{5}{|c|}{ Formulation $^{1}$} \\
\hline & Full-fat & $\begin{array}{c}\text { Traditional } \\
\text { low-fat }\end{array}$ & Prebiotic & Probiotic & Synbiotic \\
\hline \multicolumn{6}{|l|}{ Appearance } \\
\hline Color & $4.68^{\mathrm{a}}$ & $4.14^{\mathrm{a}}$ & $4.61^{\mathrm{a}}$ & $4.30^{\mathrm{a}}$ & $4.81^{\mathrm{a}}$ \\
\hline Brightness & $2.51^{\mathrm{d}}$ & $4.95^{\mathrm{c}}$ & $6.62^{\mathrm{ab}}$ & $5.55^{\mathrm{bc}}$ & $7.27^{\mathrm{a}}$ \\
\hline Syneresis & $1.60^{\mathrm{c}}$ & $1.00^{\mathrm{c}}$ & $5.12^{\mathrm{b}}$ & $1.98^{\mathrm{c}}$ & $6.78^{\mathrm{a}}$ \\
\hline Firmness & $7.39^{\mathrm{b}}$ & $7.66^{\mathrm{a}}$ & $7.51^{\mathrm{ab}}$ & $7.54^{\mathrm{ab}}$ & $7.62^{\mathrm{ab}}$ \\
\hline Homogeneity & $3.52^{\mathrm{b}}$ & $6.91^{\mathrm{a}}$ & $6.24^{\mathrm{a}}$ & $6.25^{\mathrm{a}}$ & $7.32^{\mathrm{a}}$ \\
\hline \multicolumn{6}{|l|}{ Aroma } \\
\hline Acid & $3.47^{\mathrm{b}}$ & $4.68^{\mathrm{ab}}$ & $5.09^{\mathrm{a}}$ & $5.03^{\mathrm{a}}$ & $5.64^{\mathrm{a}}$ \\
\hline Fermented milk & $2.96^{\mathrm{b}}$ & $4.59^{\mathrm{a}}$ & $4.24^{\mathrm{a}}$ & $4.39^{\mathrm{a}}$ & $5.03^{\mathrm{a}}$ \\
\hline \multicolumn{6}{|l|}{ Flavor } \\
\hline Acid taste & $5.23^{\mathrm{ab}}$ & $5.43^{\mathrm{ab}}$ & $4.78^{\mathrm{b}}$ & $6.08^{\mathrm{a}}$ & $6.14^{\mathrm{a}}$ \\
\hline Sweet taste & $2.87^{\mathrm{a}}$ & $2.39^{\mathrm{a}}$ & $2.42^{\mathrm{a}}$ & $2.22^{\mathrm{a}}$ & $2.04^{\mathrm{a}}$ \\
\hline \multicolumn{6}{|l|}{ Texture } \\
\hline Creaminess & $6.91^{\mathrm{a}}$ & $5.92^{\mathrm{b}}$ & $5.86^{\mathrm{b}}$ & $5.58^{\mathrm{b}}$ & $5.79^{\mathrm{b}}$ \\
\hline Homogeneity & $5.18^{\mathrm{c}}$ & $6.78^{\mathrm{ab}}$ & $7.09^{\mathrm{a}}$ & $5.88^{\mathrm{bc}}$ & $6.69^{\mathrm{ab}}$ \\
\hline Firmness & $5.67^{\mathrm{b}}$ & $7.04^{\mathrm{a}}$ & $6.45^{\mathrm{ab}}$ & $7.48^{\mathrm{a}}$ & $6.78^{\mathrm{a}}$ \\
\hline
\end{tabular}

${ }^{\mathrm{a} d}$ Means within a row with different superscript letters are different $(P \leq 0.05)$.

${ }^{1}$ Prebiotic $=$ low-fat yogurt with $2 \%$ long-chain inulin; probiotic $=$ low-fat yogurt with Lactobacillus paracasei ssp. paracase $;$; synbiotic $=$ low-fat yogurt with $2 \%$ long-chain inulin and Lb. paracase $i$ ssp. paracasei.

only when it is used as a partial substitute for fat; that is, when at least a small amount of fat is present in the product. In fact, Guggisberg et al. (2009) observed that for yogurts that presented from 1 to $3.5 \%$ of fat, an increase in the amount of inulin in the formulation caused an increase in the perception of creaminess, but when low-fat yogurts (fat content of $0.1 \%$ ) had inulin added to them, no influence on creaminess was perceived by consumers.

The results demonstrate that the use of inulin improves some sensory characteristics of low-fat yogurt, such as brightness and firmness. However, yogurts with added long-chain inulin presented higher syneresis and no influence on creaminess was perceived by the sensory panel.

Table 3 shows the results for the consumer test. The acceptability scores of the yogurts ranged between scores 6 and 7 on a 9-point hedonic scale, indicating that consumers slightly liked the products. Despite this narrow hedonic scale, differences $(P \leq 0.05)$ in acceptance were observed.

The removal of fat negatively affected the acceptance; the full-fat formulation was more accepted than the traditional low-fat formulation $(P \leq 0.05)$. However, the addition of inulin contributed to improving the acceptance of low-fat yogurt containing or not containing probiotics; that is, the acceptability of prebiotic and synbiotic formulations was similar to the full-fat formulation $(P>0.05$; Table 3$)$.

The results indicate that although inulin causes increased syneresis of the products and did not influence the perceived creaminess, it still can be considered as a fat replacer, making the low-fat products as accepted as the full-fat yogurts. Guven et al. (2005) also observed no difference in the overall acceptance of low-fat yogurts with $1 \%$ inulin added and full-fat yogurts. Spiegel et al. (1994) reported better acceptance of yogurt with added fructooligosaccharides compared with the control

Table 3. Acceptability of yogurts

\begin{tabular}{lcccc}
\hline Formulation $^{2}$ & $\begin{array}{c}\text { Overall } \\
\text { acceptability }\end{array}$ & $\begin{array}{c}\% \\
\text { Approval }\end{array}$ & $\begin{array}{c}\% \\
\text { Indifference }\end{array}$ & $\begin{array}{c}\% \\
\text { Rejection }\end{array}$ \\
\hline Full-fat & $6.8 \pm 1.6^{\mathrm{a}}$ & 80.00 & 5.33 & 14.67 \\
Traditional low-fat & $6.0 \pm 17^{\mathrm{b}}$ & 65.33 & 10.67 & 24.00 \\
Prebiotic & $6.2 \pm 1.7^{\mathrm{ab}}$ & 73.34 & 9.33 & 17.33 \\
Probiotic & $6.0 \pm 1.6^{\mathrm{b}}$ & 68.00 & 13.33 & 18.67 \\
Synbiotic & $6.1 \pm 1.7^{\text {ab }}$ & 66.67 & 12.0 & 21.33 \\
\hline
\end{tabular}

$\overline{a, b}$ Within a column, values with different superscript letters are significantly different $(P \leq 0.05)$.

${ }^{1}$ Hedonic scale: $1=$ dislike extremely, $9=$ like extremely; $\%$ approval $=$ percentage of scores between 6 and 9 ; $\%$ indifference $=$ percentage of scores of $5 ; \%$ rejection $=$ percentage of scores between 1 and 4 .

${ }^{2}$ Prebiotic $=$ low-fat yogurt with $2 \%$ long-chain inulin; probiotic $=$ low-fat yogurt with Lactobacillus paracasei ssp. paracase $i$; synbiotic $=$ low-fat yogurt with $2 \%$ long-chain inulin and $L b$. paracase $i$ ssp. paracasei. 


\section{Correlations on axes $\mathrm{t} 1$ and $\mathrm{t} 2$}

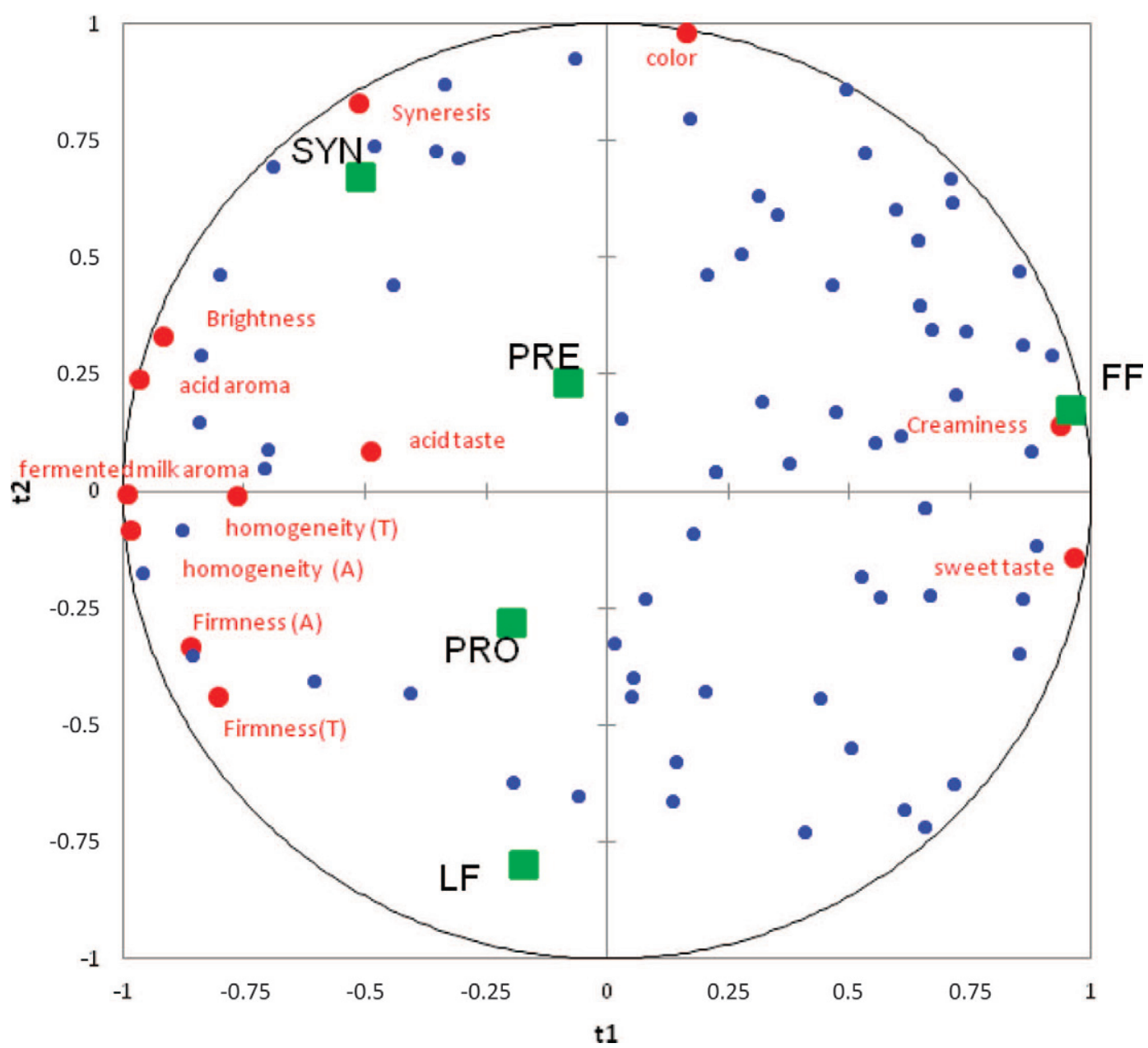

Figure 2. External preference mapping. Square $=$ samples; circle $=$ consumers. SYN $=$ synbiotic (low-fat yogurt with added inulin and Lactobacillus paracasei ssp. paracasei); PRE = prebiotic (low-fat yogurt with added inulin); PRO = probiotic (low-fat yogurt with added Lactobacillus paracasei ssp. paracasei); FF = full-fat yogurt; $\mathrm{LF}=$ traditional low-fat yogurt. An $\mathrm{A}$ in parentheses corresponds to attributes evaluated for appearance and a $\mathrm{T}$ in parentheses corresponds to attributes evaluated for texture. Color version available in the online PDF.

yogurt, and the inulin-type fructan promoted higher acceptance of appearance, texture, and creaminess of the products.

Comparing the low-fat formulations (traditional, prebiotic, probiotic, and synbiotic), no difference was observed in the sensory acceptance among them; therefore, the addition of the probiotic agent did not affect the acceptance, although the percentage of rejection was higher for the traditional low-fat formulation (24\%) compared with the formulation with added probiotic (18\%; Table 3). Similar results were observed in the acceptability of conventional and probiotic yogurts (Kailasapathy, 2006; Marafon et al., 2011; Akalın et al., 2012; Cruz et al., 2012a).

Figure 2 shows the results for the external preference mapping. Using 2 dimensions, it was possible to explain $84.9 \%$ of the variance in sensory descriptors obtained from QDA, and a total of $57.3 \%$ of the variance on the sensory acceptance. The full-fat yogurt presented the highest acceptance due to its higher perception of sweetness and creaminess, which are the characteristics that contributed positively to the acceptance of the product. On the other hand, the traditional low-fat yogurt was the least preferred sample, probably due to its higher firmness and homogeneity. Prebiotic, probiotic, and synbiotic samples were characterized by being very acidic, firm, and homogeneous, besides presenting fermented milk aroma, acid taste, and brightness. The results obtained by external preference mapping demonstrate the importance of sweetness and texture (creaminess) on the acceptance of yogurts.

In conclusion, the addition of long-chain inulin in low-fat yogurt improves the brightness and decreases its firmness, making it similar to the traditional full-fat 
product and, with respect to acceptability, makes lowfat yogurts as well accepted sensorially as the full-fat product, confirming the role of long-chain inulin as a fat replacer. However, the addition of inulin in low-fat yogurt increases the syneresis and no significant change in creaminess was perceived by the sensory panel. The addition of $L b$. paracasei ssp. paracase $i$, in turn, results in no changes in the acceptability and sensory profile of low-fat yogurt.

It is possible to formulate low-fat yogurts with added probiotics that present similar sensory characteristics compared with full-fat yogurts due to the addition of the long-chain inulin as fat replacer. However, in order to obtain a better acceptance of products, significant improvements should be performed to optimize the product's firmness (appearance and texture) and homogeneity (appearance), which were considered the drivers of disliking of samples.

\section{REFERENCES}

Akalin. A. S., and E. Erișir. 2008. Effects of inulin and oligofructose on the rheological characteristics and probiotic culture survival in low-fat probiotic ice cream. J. Food Sci. 73:M184-M187.

Akalın, A. S., S. Fenderya, and N. Akbulut. 2004. Viability and activity of bifidobacteria in yoghurt containing fructooligosaccharide during refrigerated storage. Int. J. Food Sci. Technol. 39:613-621.

Akalin, A. S., G. Gönç, G. Ünal, and S. Fenderya. 2007. Effects of fructooligosaccharide and whey protein concentrate on the viability of starter culture in reduced-fat probiotic yogurt during storage. J. Food Sci. 72:M222-M227.

Akalın, A. S., G. Unal, N. Dinkci, and A. A. Hayaloglu. 2012. Microstructural, textural, and sensory characteristics of probiotic yogurts fortified with sodium calcium caseinate or whey protein concentrate. J. Dairy Sci. 95:3617-3628.

Cadena, R. S., A. G. Cruz, J. A. F. Faria, and H. M. A. Bolini. 2012. Reduced fat and sugar vanilla ice creams: Sensory profiling and external preference mapping. J. Dairy Sci. 95:4842-4850.

Chapman, K. W., H. T. Lawless, and K. J. Boor. 2001. Quantitative descriptive analysis and principal component analysis for sensory characterization of ultrapasteurized milk. J. Dairy Sci. 84:12-20.

Cieślik, E., A. Kopeć, and P. M. Pisulewski. 2005. Effect of fructooligosaccharides and long-chain inulin on serum lipids in rats. Pol. J. Food Nutr. Sci. 14:437-441.

Coudray, C., C. Feillet-Coudray, J. C. Tressol, E. Gueux, S. Thien, L. Jaffrelo, A. Mazur, and I. Rayssuguier. 2005. Stimulatory effect of inulin on intestinal absorption of calcium and magnesium in rats is modulates by dietary intakes. Eur. J. Nutr. 44:293-302.

Cruz, A. G., R. S. Cadena, J. A. F. Faria, H. M. A. Bolini, C. Dantas, M. M. C. Ferreira, and R. Deliza. 2012a. PARAFAC: Adjustment for modeling consumer study covering probiotic and conventional yogurt. Food Res. Int. 45:211-215.

Cruz, A. G., W. F. Castro, J. A. F. Faria, P. C. B. Lollo, J. AmayaFarfán, M. Q. Freitas, D. Rodrigues, C. A. F. Oliveira, and H. T. Godoy. 2012b. Probiotic yogurts manufactured with increased glucose oxidase levels: Postacidification, proteolytic patterns, survival of probiotic microorganisms, production of organic acid and aroma compounds. J. Dairy Sci. 95:2261-2269.

Cruz, A. G., R. N. Cavalcanti, L. M. R. Guerreiro, A. S. Sant'ana, L. C. Nogueira, C. A. F. Oliveira, R. Deliza, R. L. Cunha, J. A. F. Faria, and H. M. A. Bolini. 2013. Developing a prebiotic yogurt: Rheological, physico-chemical and microbiological aspects and adequacy of survival analysis methodology. J. Food Eng. 114:323-330.
Damasio, M. H., and E. Costell. 1991. Análisis sensorial descriptivo: Generatión de descriptores y selección de catadores. Revista de Agroquimica y Tecnologia de Alimentos 31:165-178.

Den Hond, E., B. Geypens, and Y. Ghoos. 2000. Effect of high performance chicory inulin on constipation. Nutr. Res. 20:731-736.

Donkor, O. N., S. L. I. Nilmini, P. Stolic, T. Vasiljevic, and N. P. Shah. 2007. Survival and activity of selected probiotic organisms in set-type yoghurt during cold storage. Int. Dairy J. 17:657-665.

Drake, M. A. 2007. Invited review: Sensory analysis of dairy foods. J Dairy Sci. 90:4925-4937.

FAO/AGNS (Food and Agriculture Organization of the United $\mathrm{Na}-$ tions/Food Quality and Standards Service). 2007. FAO Technical Meeting Report on Prebiotics. AGNS and FAO, Rome, Italy.

FAO/WHO (Food and Agriculture Organization of the United Nations/World Health Organization). 2001. Health and nutritional properties of probiotics in food including powder milk with live lactic acid bacteria. Report of a joint FAO/WHO expert consultation, Córdoba, Argentina. Accessed Jul. 29, 2013. ftp://ftp.fao. org/docrep/fao/meeting/009/y6398e.pdf.

Franck, A. 2002. Technological functionality of inulin and oligofructose. Br. J. Nutr. 87(Suppl. 2):S287-S291.

Gonzalez, N. J., K. Adhikari, and M. F. Sancho-Madriz. 2011. Sensory characteristics of peach-flavored yogurt drinks containing prebiotics and synbiotics. Lebensm. Wiss. Technol. 44:158-163.

Gonzalez-Tomás, L., J. Coll-Marqués, and E. Costell. 2008. Viscoelasticity of inulin-starch-based dairy systems. Influence of inulin average chain length. Food Hydrocoll. 22:1372-1380.

Granato, D., I. A. de Castro, L. S. N. Ellendersen, and M. L. Masson. 2010. Physical stability assessment and sensory optimization of a dairy-free emulsion using response surface methodology. J. Food Sci. 75:S149-S155.

Guggisberg, D., J. Cuthbert-Steven, P. Picccinali, U. Bütikofer, and P. Eberhard. 2009. Rheological, microstructural and sensory characterization of low-fat and whole milk set yoghurt as influenced by inulin addition. Int. Dairy J. 19:107-115.

Guven, M., K. Yasar, O. B. Karaca, and A. A. Hayaloglu. 2005. The effect of inulin as a fat replacer on the quality of set-type low-fat yogurt manufacture. Int. J. Dairy Technol. 58:180-184.

Hair, J. F., Jr., W. C. Black, B. J. Babin, and R. E. Anderson. 2010. Multivariate Data Analysis. Pearson Prentice Hall, Upper Saddle River, NJ.

Hennelly, P. J., P. G. Dunne, M. O'Sullivan, and E. D. O'Riordan. 2006. Textural, rheological and microstructural properties of imitation cheese containing inulin. J. Food Eng. 75:388-395.

Hughes, R., and I. R. Rowland. 2001. Stimulation of apoptosis by two prebiotic chicory fructans in the rat colon. Carcinogenesis 22:43-47.

Kaaki, D., O. Kebbe Baghdadi, N. E. Najm, and A. Olabi. 2012. Preference mapping of commercial Labneh (strained yogurt) products in the Lebanese market. J. Dairy Sci. 95:521-532.

Kailasapathy, K. 2006. Survival of free and encapsulated probiotic bacteria and their effect on the sensory properties of yoghurt. Lebensm. Wiss. Technol. 39:1221-1227.

Karaca, O. B., M. Güven, K. Yasar, S. Kava, and T. Kahyaoglu. 2009. The functional, rheological and sensory characteristics of ice creams with various fat replacers. Int. J. Dairy Technol. 62:93-99.

Koca, N., and M. Metin. 2004. Textural, melting and sensory properties of low-fat fresh Kashar cheeses produced by using fat replacers. Int. Dairy J. 14:365-373.

Lawless, H. T., and H. Heymann. 1998. Sensory Evaluation of Food: Principles and Practices. Chapman \& Hall, New York, NY.

Liu, C.-T., F.-J. Chu, C.-C. Chou, and R.-C. Yu. 2011. Antiproliferative and anticytotoxic effects of cell fractions and exopolysaccharides from Lactobacillus casei 01. Mutat. Res. 721:157-162.

Marafon, A. P., A. Sumi, D. Granato, M. R. Alcântara, A. Y. Tamime and M. N. de Oliveira. 2011. Effects of partially replacing skimmed milk powder with dairy ingredients on rheology, sensory profiling, and microstructure of probiotic stirred-type yogurt during cold storage. J. Dairy Sci. 94:5330-5340.

Meilgaard, M., G. V. Civille, and B. T. Carr. 1998. Sensory Evaluation Techniques. 3rd ed. CRC Press, London, UK. 
Meyer, D., S. Bayarri, A. Tárrega, and E. Costell. 2011a. Inulin as texture modifier in dairy products. Food Hydrocoll. 25:1881-1890.

Meyer, D., J. Vermulst, R. H. Tromp, and E. H. A. de Hoog. 2011b. The effect of inulin on tribology and sensory profiles of skimmed milk. J. Texture Stud. 42:387-393.

Mirzaei, H., B. A. Tabrizi, A. Hasanpour, A. Babapour, and G. Karim. 2008. Study on the effect of consuming different amount of fermented milk with Lactobacillus casei 01 on haematological parameters in rats. Res. J. Biol. Sci. 3:1376-1380.

Modzelewska-Kapituła, M., and L. Kłębukowska. 2009. Investigation of the potential for using inulin HPX as a fat replacer in yoghurt production. Int. J. Dairy Technol. 62:209-214.

Moskowitz, H. R. 1983. Product Testing and Sensory Evaluation of Food: Marketing and R\&D Approaches. Food and Nutrition Press, Westport, CT.

Paseephol, T., D. M. Small, and F. Sherkat. 2008. Rheology and texture of set yogurt as affected by inulin addition. J. Texture Stud. 39:617-634.

Patterson, J. K., K. Yasuda, R. M. Welch, D. D. Miller, and X. G. Le. 2010. Supplemental dietary inulin of variable chain lengths alters intestinal bacterial populations in young pigs. J. Nutr. 140:2158-2161.

Pimentel, T. C., S. Garcia, and S. H. Prudencio. 2012a. Aspectos funcionais, de saúde e tecnológicos de frutanos tipo inulina. Boletim do Centro de Pesquisa de Processamento de Alimentos 30:103118
Pimentel, T. C., S. Garcia, and S. H. Prudencio. 2012b. Effect of longchain inulin on the texture profile and survival of Lactobacillus paracasei ssp. paracasei in set yoghurts during refrigerated storage. Int. J. Dairy Technol. 65:104-110.

Ryz, N. R., J. B. Meddings, and C. G. Taylor. 2009. Long-chain inulin increases dendritic cells in the Peyer's patches and increases ex vivo cytokine secretion in the spleen and mesenteric lymph nodes of growing female rats, independent of zinc status. Br. J. Nutr. 101:1653-1663.

Spiegel, J. E., R. Rose, P. Karabell, V. H. Frankos, and D. F. Schmitt. 1994. Safety and benefits of fructooligosaccharides as food ingredients. Food Technol. 48:85-89.

Tharmaraj, N., and N. P. Shah. 2009. Antimicrobial effects of probiotics against selected pathogenic and spoilage bacteria in cheesebased dips. Int. Food Res. J. 16:261-276.

Torres, J. D., A. Tárrega, and E. Costell. 2010. Storage stability of starch-based dairy desserts containing long-chain inulin: Rheology and particle size distribution. Int. Dairy J. 20:46-52

Villegas, B., I. Carbonell, and E. Costell. 2007. Inulin milk beverages: Sensory differences in thickness and creaminess using R-index analysis of the ranking data. J. Sens. Stud. 22:377-393.

Wada, T., J. N. Sugatani, E. Terada, M. Ohguchi, and M. Miwa. 2005. Physicochemical characterization and biological effects of inulin enzymatically synthesized from sucrose. J. Agric. Food Chem. $53: 1246-1253$ 